\title{
Changes in neurophysiologic markers of visual processing following beneficial anti-VEGF treatment in macular degeneration
}

This article was published in the following Dove Press journal:

Clinical Ophthalmology

26 February 2013

Number of times this article has been viewed

\author{
Pasi Vottonen' \\ Kai Kaarniranta ${ }^{1,2}$ \\ Ari Pääkkönen ${ }^{3}$ \\ Ina M Tarkka ${ }^{4}$ \\ 'Department of Ophthalmology, \\ Kuopio University Hospital, \\ Kuopio, Finland; ${ }^{2}$ Department of \\ Ophthalmology, Institute of Clinical \\ Medicine, University of Eastern \\ Finland, Kuopio, Finland; ${ }^{3}$ Department \\ of Clinical Neurophysiology, Institute \\ of Clinical Medicine, University of \\ Eastern Finland, Kuopio, Finland; \\ ${ }^{4}$ Department of Health Sciences, \\ University of Jyväskylä, Jyväskylä, \\ Finland
}

Purpose: Antivascular endothelial growth factor (VEGF) agents have been shown to improve visual acuity and prevent vision loss in exudative age-related macular degeneration. As the vision improves relatively quickly in response to intravitreal injections, we wanted to know whether this improvement is reflected in electrophysiological markers of visual cortical processing.

Patients and methods: Our interventional case series included six elderly patients who underwent injection treatment to the affected eye. Their visual acuity, tomographic images of retinal thickness, and visual evoked potentials (VEP) were assessed before treatment and six weeks after the last injection.

Results: All patients showed improved visual acuity and reduced retinal fluid after the treatment. All but one patient showed increased VEP P100 component amplitudes and/or shortened latencies in the treated eye. These VEP changes were consistent with improved vision while the untreated eyes showed no changes.

Conclusions: Our results indicate that antivascular endothelial growth factor injections improved visual function of the treated eyes both in the level of the retina and in the level of visual cortical processing.

Keywords: age-related eye diseases, exudative age-related macular degeneration, visual evoked potentials, scalp-recorded EEG, visual acuity

\section{Introduction}

Age-related macular degeneration (AMD) is the major cause of central blindness in the elderly in Western countries. It is characterized by a progressive loss of color and fine vision, reduced contrast, and spatiotemporal sensitivity.

Clinically, AMD can be classified into early- or late-atrophic AMD (ie, dry AMD), or exudative (ie, wet) AMD. ${ }^{1}$ Wet AMD is characterized by proliferation of choroidal neovascularization. Excessive expression of vascular endothelial growth factor (VEGF) by retinal pigment epithelial (RPE) cells leads to new vessel formation originating from the choroid and extending through defects in the Bruch's membrane and the RPE sub- and intraretinally. ${ }^{2}$ Vision loss occurs through the structural and metabolic damage in the retina and in the RPE cells. ${ }^{1-3}$ To date, anti-VEGF (bevacizumab or ranibizumab) intravitreal injections have been shown to be an effective treatment to decrease retina fluid in exudative AMD., ${ }^{4,5}$ Vision improvement occurs within 1 month and continues to improve over the next few months.

Optical coherence tomography (OCT) is a standard technique for cross-sectional imaging of the retina. OCT is useful in quantitatively evaluating subretinal and intraretinal fluid, assessing possible subfoveal involvement of neovascularization, and in
Correspondence: Kai Kaarniranta Department of Ophthalmology, Kuopio University Hospital, PO Box 1777, 702II Kuopio, Finland Tel +35817172485

Fax +35817172485

Email kai.kaarniranta@uef.fi 
monitoring anti-VEGF intravitreal injection response. Visual evoked potentials (VEP) provide quantitative information on the functional integrity of well-defined visual pathways in the central nervous system. For instance, for analysis of the visual pathways, VEP are considered more sensitive than magnetic resonance imaging in detecting optic neuritis and clinically silent lesions in multiple sclerosis. ${ }^{6}$

We aimed to investigate whether the improved vision by anti-VEGF treatment is reflected in the function of the visual pathways. Our hypothesis was that significant changes in VEP will be observed, and these changes may be related both to improvement in vision and decreased retinal fluid.

\section{Materials and methods}

The interventional case series consisted of six patients having exudative AMD. Diagnosis was based on the best corrected visual acuity (BCVA) testing, full biomicroscopy examination and OCT (Spectral OCT/SLO, Ophthalmic Technologies Inc, Toronto, ON, Canada) analysis in the Department of Ophthalmology, Kuopio University Hospital. For patient characteristics and their visual acuities see Table 1. At the first visit, when exudative AMD diagnosis was made, bevacizumab intravitreal injection was applied into the affected eye of each individual. The injection was performed three times, 6 weeks between injections. VEP, BCVA testing, full biomicroscopy analysis, and OCT examinations were performed at the first visit prior to anti-VEGF injection and 6 weeks after the last (ie, third) injection, about 18 weeks after the first visit. The Ethics Committee of the Kuopio University Hospital had approved the study, and the tenets of the Declaration of Helsinki were followed. All participants provided informed consent.

Visual evoked potentials were recorded using a 64-channel electrode cap (EASYCAP GmbH, Falk-Minow Services, Ammersee, Germany) with the SynAmps2 amplifier (NeuroScan, Inc, El Paso, TX, USA). All scalp electrodes

Table I Patient characteristics and their visual acuity before and after anti-VEGF treatment

\begin{tabular}{|c|c|c|c|c|c|c|}
\hline & \multirow[t]{2}{*}{ Gender } & \multirow[t]{2}{*}{ Age } & \multicolumn{2}{|c|}{ BCVA treated eye } & \multicolumn{2}{|c|}{ BCVA nontreated eye } \\
\hline & & & $\begin{array}{l}\text { Before } \\
\text { injection }\end{array}$ & $\begin{array}{l}\text { After } \\
\text { injection }\end{array}$ & $\begin{array}{l}\text { Before } \\
\text { injection }\end{array}$ & $\begin{array}{l}\text { After } \\
\text { injection }\end{array}$ \\
\hline $\mathrm{PI}$ & $\mathrm{F}$ & 78 & 0.2 & 0.7 & 0.7 & 0.7 \\
\hline $\mathrm{P} 2$ & $M$ & 90 & 0.2 & 0.5 & 0.5 & 0.5 \\
\hline P3 & $\mathrm{F}$ & 74 & 0.05 & 0.3 & 0.9 & 0.9 \\
\hline P4 & $\mathrm{F}$ & 86 & 0.5 & 0.7 & 0.8 & 0.8 \\
\hline P5 & $M$ & 75 & 0.3 & 0.5 & 1.0 & 0.9 \\
\hline P6 & $M$ & 79 & 0.2 & 0.4 & 0.8 & 0.5 \\
\hline
\end{tabular}

Abbreviations: VEGF, vascular endothelial growth factor; $P$, patient number; $\mathrm{F}$, female; M, male; BCVA, best corrected visual acuity (Snellen equivalents). were referred to a midline centrofrontal electrode $(\mathrm{FCz})$. Potentials reflecting eye blinks and vertical eye movements were recorded between electrodes placed above and below the right eye. All electrode impedances were kept below $10 \mathrm{k} \Omega$. Signals were amplified and filtered with a bandpass of $0.1-50 \mathrm{~Hz}$ and digitized continuously at $500 \mathrm{~Hz}$.

Pattern reversal VEP were elicited by a high contrast black and white checkerboard stimulus on a computer monitor using STIM2 software (NeuroScan, Inc). To cover the macular area with the stimulus, a circular stimulus field with a diameter of 10.3 degrees was used. The check size was 35 minutes of arc. The pattern was reversed at $1 \mathrm{~Hz}$. A small red fixation target was positioned at two check widths above the center of the pattern stimulus. The viewing distance was $100 \mathrm{~cm}$. Stimulation was monocular after occlusion of the other eye. For each eye and each recording session, responses to two blocks of 120 stimuli were recorded, with an undilated pupil under full refractive correction. Both in the pre- and posttreatment sessions, the eye that was not affected by wet AMD was recorded first. Each patient could clearly perceive the fixation mark with the nonaffected eye. If the patient could not see the fixation mark with the affected eye, he/she was asked to fixate at its known location.

The VEP data were processed offline with SCAN software (NeuroScan, Inc). First, the continuous data from two recording blocks were merged into one file. Then the data were filtered (high pass $1 \mathrm{~Hz}$ ) and segmented to epochs of $350 \mathrm{~ms}$ including a prestimulus interval of $50 \mathrm{~ms}$. Epochs containing blinks or other artifacts were manually rejected. The artifact-free epochs were then averaged separately for each eye and each recording session. In averaging, both the mean and standard deviation were calculated on a pointby-point basis. Responses at electrode $\mathrm{Oz}$ were chosen for further analysis.

To test the statistical significance of the changes in VEP between the pre- to posttreatment recordings in each patient, Student's $t$-test was applied to each time point of the VEP waveform. The level of statistical significance was set at $P<0.05$. To reduce the probability of interpreting chance fluctuations as true changes, we set an additional requirement for a significant change: the probability level had to be $<0.05$ at least in four consecutive data points or at least over $8 \mathrm{~ms}$.

\section{Results}

All patients showed improved vision in BCVA after the bevacizumab intravitreal treatment period (Table 1). This was in line with decreased retinal fluid (Figure 1 and Table 2). 

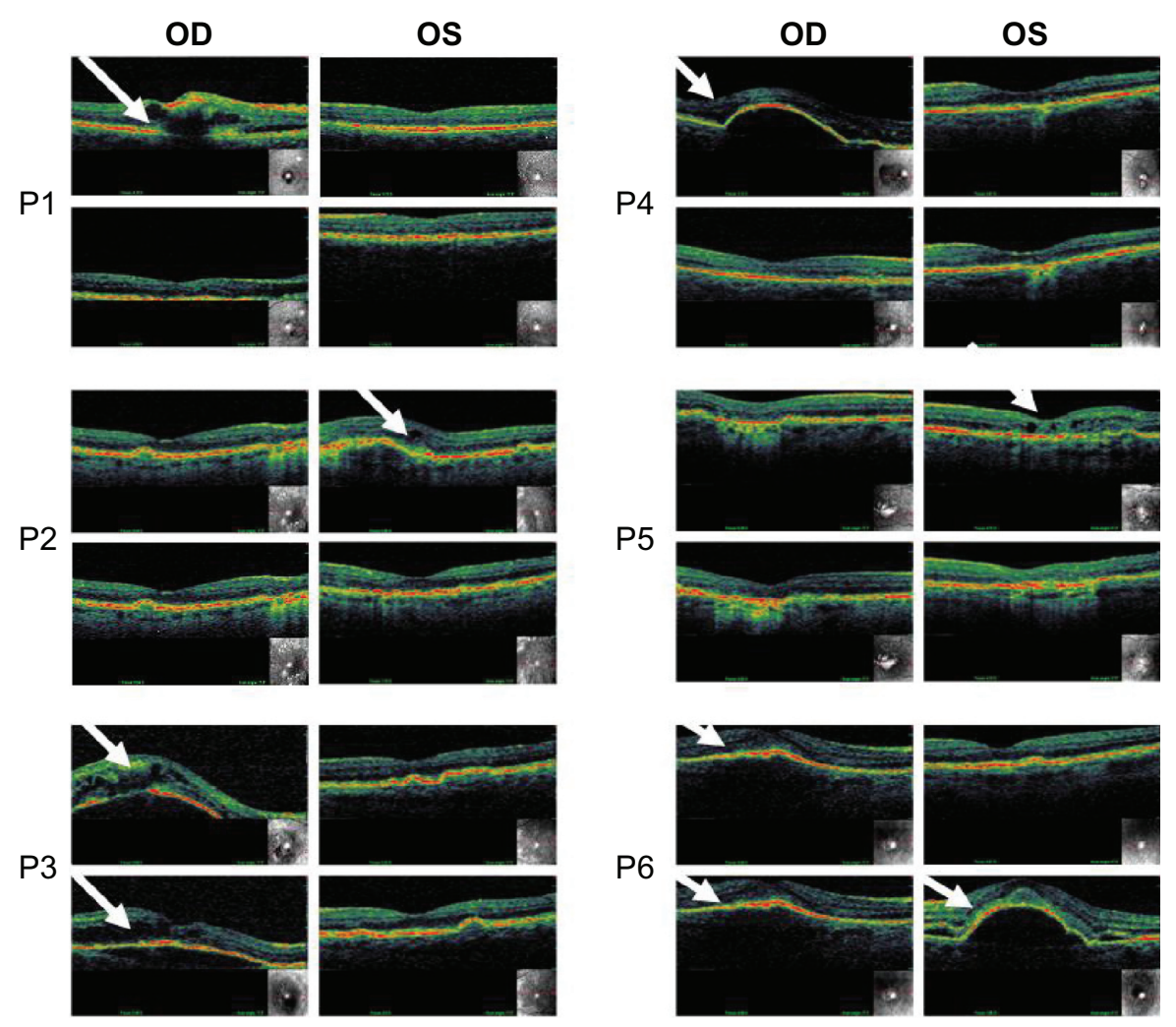

Figure I Optical coherence tomographic (OCT) images illustrating retinal thickness of each individual patient (PI-P6) before and after injections (I8 weeks in between images).

Notes: For each patient, the top row is before treatment and the bottom row is after treatment. White arrows point to the treated eye and defected sites in the retina. Note that the left eye for $\mathrm{P} 6$ was not treated during the study period.

Abbreviations: $P$, patient; OD, right eye; OS, left eye.

Each patient's individual images of optical coherence tomography are shown in Figure 1. For one of the patients (P6), the primarily atrophic AMD changed to exudative form in the left eye during the study period (Figure 1). This exudative form correlated well with decreased visual acuity and increased retinal thickness (Figure 1 and Table 1). Note that anti-VEGF therapy had only partial improvement for P3

Table 2 Retinal thickness $(\mu M)$ in individual patients measured with optical coherence tomography before and after injection in the treated and nontreated eyes

\begin{tabular}{|c|c|c|c|c|}
\hline & \multicolumn{2}{|c|}{ Treated eye } & \multicolumn{2}{|c|}{ Nontreated eye } \\
\hline & $\begin{array}{l}\text { Before } \\
\text { injection }\end{array}$ & $\begin{array}{l}\text { After } \\
\text { injection }\end{array}$ & $\begin{array}{l}\text { Before } \\
\text { injection }\end{array}$ & $\begin{array}{l}\text { After } \\
\text { injection }\end{array}$ \\
\hline PI & 536 & 240 & 253 & 253 \\
\hline P2 & 330 & 240 & 250 & 250 \\
\hline P3 & 510 & 430 & 250 & 250 \\
\hline P4 & 440 & 240 & 240 & 240 \\
\hline P5 & 400 & 230 & 250 & 240 \\
\hline P6 & 420 & 263 & 263 & 440 \\
\hline
\end{tabular}

Abbreviation: $P$, patient number. and P6, in whom the reduction in retinal thickness observed in OCT was moderate (Figure 1 and Table 2).

In five out of the six patients, posttreatment VEP waveforms indicated statistically significant changes compared to pretreatment waveforms, and these changes were consistent with improved vision in the treated eye. The VEP of the treated and untreated eyes of each patient before and after anti-VEGF treatment are illustrated in Figure 2. The changes observed in P100 were decreases in latency and/or increases in amplitude. The time periods of the significant changes in the P100 component, which are consistent with improved vision, are marked with dark grey bars. All these changes were observed in the treated eye. Additional time periods with significant changes were observed outside the target component P100, and they are marked with transparent bars (Figure 2). Those VEP changes were observed mainly in the eyes in which the treatment effect on P100 was significant, indicating that the whole VEP waveform may be affected. Slowing and/or reduced P100 amplitude was observed in the untreated eye in four out of six cases. The overall result 
Treated eye

P1

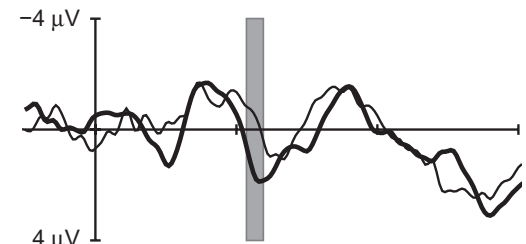

P2

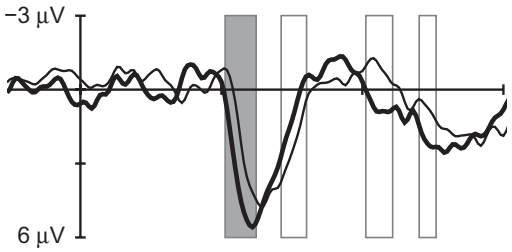

P3

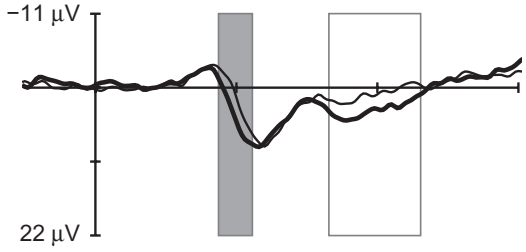

P4

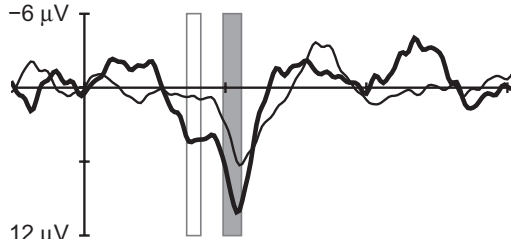

P5

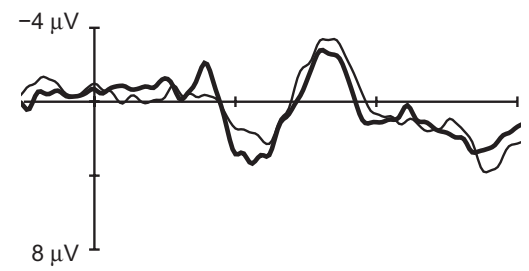

P6

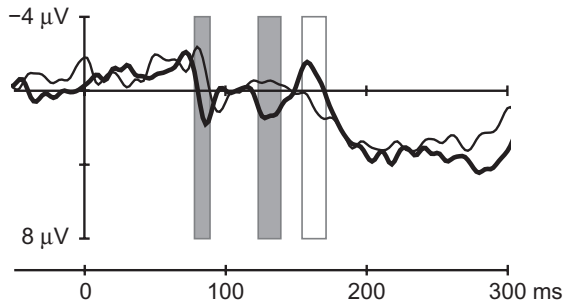

Untreated eye
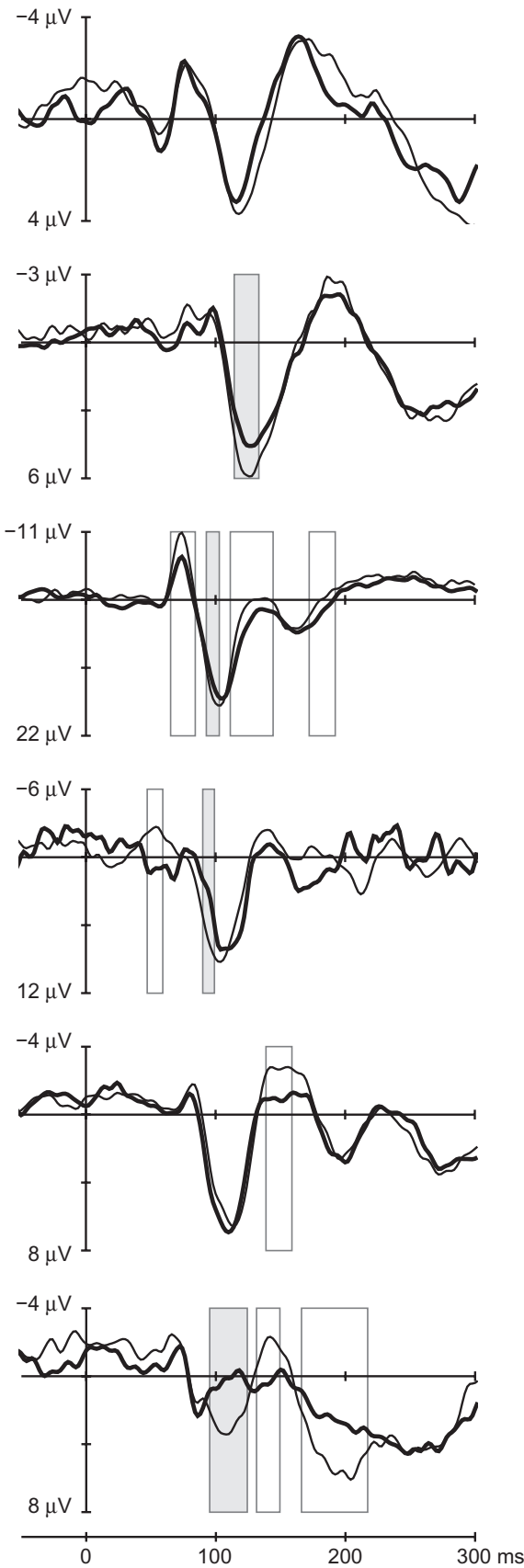

Figure 2 VEP in the Oz-FCz derivation before (thin line) and after (thick line) anti-VEFG treatment from the treated and nontreated eyes of six patients.

Notes: The waveforms are the average of the about 240 sweeps presented in the two trials. The bars indicate the time ranges over which the curves differ statistically at the probability level of $P<0.05$ at least over $8 \mathrm{~ms}$. The dark grey bars correspond to changes consistent with improved vision (ie, with a decrease in PI00 latency and/or an increase in PI00 amplitude), whereas the light bars correspond to opposite changes. The white bars correspond to changes outside the PI00 component. In $5 / 6$ patients, statistically significant changes consistent with improved vision in the treated eye were observed. Note that in P6, there had been a deterioration of vision in the untreated eye between the recordings leading to large changes in VEP from that eye.

Abbreviations: VEP, visual evoked potentials; $P$, patient number.

in VEP is illustrated in Figure 3, where the grand averaged waveforms of all six patients in the $\mathrm{Oz}$ scalp location are shown. Remarkably, the latency decrease was in the order of $7 \mathrm{~ms}$, and the amplitude increase in the order of $30 \%$ was observed at 18 weeks in elderly patients.

\section{Discussion}

Intravitreal injection of bevacizumab appears to result in significant improvements in visual acuity and a reduction of retinal thickness secondary to exudative AMD. ${ }^{4,5,7}$ The present results are in line with those of multicenter trials 
Treated eye - grand average $(\mathrm{N}=6)$

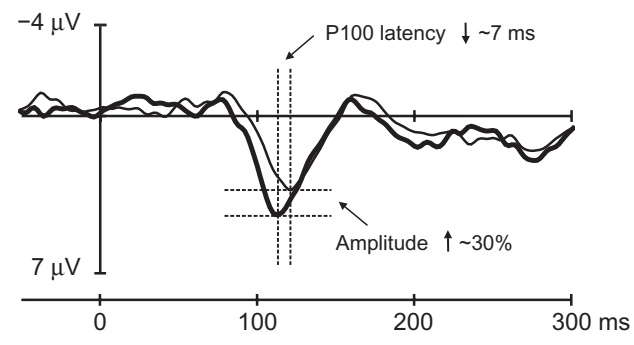

Untreated eye - grand average $(\mathrm{N}=5)$

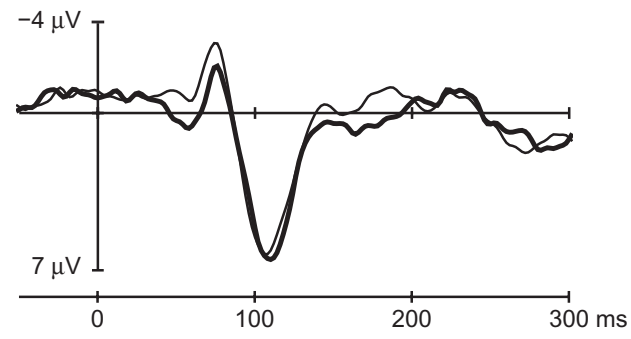

Figure 3 Grand average waveforms of individual waveforms from Figure 2.

Notes: The improvement due to the treatment is reflected by the shortening of the PI00 latency by about 7 ms and by its amplitude increase of about $30 \%$. Due to a small number of subjects in the grand average and their large individual variation, the spread of the average data is so large that the curves and the numbers presented are only for illustration and have no statistical significance.

on the effect of bevacizumab in the treatment of exudative AMD. No ocular toxicity or adverse effects were observed in our patients for short-term bevacizumab treatment. Bevacizumab is well tolerated, as was shown in the previous large studies. ${ }^{4,5,7}$ It is not known why some patients do not respond fully to anti-VEGF therapy. In the present study, the cases P3 and P6 showed only limited response to bevacizumab. AMD is a multifactorial disease that affects the aging process of the RPE cells and their adjacent tissues, and this may play a role in the individual response to treatment. ${ }^{3}$ In addition, the delay to receive intravitreal anti-VEGF injection during active exudative AMD process may diminish treatment results. ${ }^{7}$ That may be the reason behind the results for cases P3 and P6.

A remarkable decrease in P100 latency and increase in amplitude was observed in the treated eye, but no changes were observed in the untreated eye in the VEP study. The changes in the VEP are most likely due to changes at the macular level. However, if there were no processing changes at all in the higher levels of the visual system, one would expect that in cases in which a large part of the macula stays intact there would be no delay in the onset of the P100 component. Our results (Figure 2, Patients 1, 2, 3, and 6) show otherwise. Therefore we suggest that the changes in VEP are secondary to changes in the macula. We do however say that VEP is a neurophysiological marker of visual processing. Until now, the effects of intravitreal treatment of AMD in cortical visual processing are largely unknown. In one study of 21 vein occlusion patients successfully treated with bevacizumab injections, VEP were measured only in two patients before injection and at 4 weeks without any significant changes in VEP. ${ }^{8}$ However, few reports of the possible changes in the function of visual pathways in diabetes exist. A VEP study using multifocal stimulation (mfVEP) in adult subjects with diabetes showed significantly delayed
VEP compared to nondiabetic controls. ${ }^{9}$ Diabetic subjects with or without retinopathy demonstrated this delay. This suggests that retinopathy-free patients are showing early neurodegenerative changes occurring either at the level of the retina or upstream in the visual pathway that contribute to a cortical visual response. The mfVEP amplitude measurement appeared to be less sensitive to the effects of diabetes than the latency measurement. Furthermore, a specific analysis of mfVEP indicated that zones with retinopathy showed significantly delayed VEP compared to zones without retinopathy according to Wolff et al. ${ }^{9}$ Even in patients without retinopathy, the mfVEP is able to detect preclinical microvascular and/or neurodegenerative changes within or upstream from the retina. Recently a VEP study was also performed in 45 children, 15 with recently discovered juvenile diabetes (DMI), 15 children with long-lasting DMI, and 15 healthy children. Amplitude values decreased progressively, and latency values increased progressively in children with DMI as the years passed. ${ }^{10}$

Thus VEP can detect early signs of diminishing visual ability; however, we were able to detect encouraging VEP modulation within 18 weeks in elderly patients who received intravitreal treatment for AMD. In this study, in most treated eyes, the latencies of P100 were shortened. Since the VEP is covering the whole area of the macula (the healthy part and not only the AMD affected area), the changes of P100 latencies were surprisingly long. One could assume that the healthy part of the macula would produce the signals with normal latencies, and only the affected area would produce longer P100 latencies. It also seems that signals from the healthy part are delayed. This can be interpreted in a way that suggests that there are changes in the visual pathway, and not only in the retina despite the improved vision. Since no VEP changes toward the same direction were observed in the nontreated eye, the finding supports the concept that 
improved peripheral input plays a significant role in the neural plasticity of the visual cortex. In order to confirm these findings, larger population studies are required, and functional technological analyses are needed. ${ }^{11,12}$

\section{Acknowledgments}

This work was supported by the Academy of Finland, the EVO grant 5503726, the Finnish Cultural Foundation and its North Savo Fund, the Finnish Eye Foundation, the Finnish Funding Agency for Technology and Innovation, and the Päivikki and Sakari Sohlberg Foundation.

\section{Author contributions}

Pasi Vottonen: clinical examination, manuscript writing; Kai Kaarniranta: study planning, clinical examination, manuscript writing, economical support; Ari Pääkkönen: study planning, manuscript writing; Ina Tarkka: study planning, manuscript writing.

\section{Disclosure}

The authors report no conflicts of interest in this work.

\section{References}

1. Gehrs KM, Anderson DH, Johnson LV, Hageman GS. Age-related macular degeneration - emerging pathogenetic and therapeutic concepts. Ann Med. 2006;38(7):450-471.

2. Salminen A, Kauppinen A, Hyttinen JM, Toropainen E, Kaarniranta K. Endoplasmic reticulum stress in age-related macular degeneration: trigger for neovascularization. Mol Med. 2010;16(11-12):535-542.
3. Kaarniranta K, Salminen A, Haapasalo A, Soininen H, Hiltunen M. Age-related macular degeneration (AMD): Alzheimer's disease in the eye? J Alzheimers Dis. 2011;24(4):615-631.

4. Chakravarthy U, Harding SP, Rogers CA, et al; for IVAN Study Investigators. Ranibizumab versus bevacizumab to treat neovascular age-related macular degeneration: one-year findings from the IVAN randomized trial. Ophthalmology. 2012;119(7):1399-1411.

5. Martin DF, Maguire MG, Ying GS, et al; for CATT Research Group. Ranibizumab and bevacizumab for neovascular age-related macular degeneration. N Engl J Med. 2011;364(20):1897-1908.

6. Ko KF. The role of evoked potential and MR imaging in assessing multiple sclerosis: a comparative study. Singapore Med J. 2010;51(9): 716-720.

7. Rosenfeld PJ, Brown DM, Heier JS, et al; for MARINA Study Group. Ranibizumab for neovascular age-related macular degeneration. $N E n g l$ J Med. 2006;355(14):1419-1431.

8. Pai SA, Shetty R, Vijayan PB, et al. Clinical, anatomic, and electrophysiologic evaluation following intravitreal bevacizumab for macular edema in retinal vein occlusion. Am J Ophthalmol. 2007;143(4):601-606.

9. Wolff BE, Bearse MA Jr, Schneck ME, Barez S, Adams AJ. Multifocal VEP (mfVEP) reveals abnormal neuronal delays in diabetes. Doc Ophthalmol. 2010;121(3):189-196.

10. Karlica D, Galetović D, Bućan K, Znaor L. Retinoscopy and visual evoked potential based evaluation of the exactness of hyperopia correction in children with developmental difficulties. Acta Clin Croat. 2010;49(3):251-255.

11. Macky TA, Mahgoub MM. Electrophysiological assessment of optic nerve and retinal functions following intravitreal injection of bevacizumab (avastin). J Ocul Pharmacol Ther. 2012;28(2):159-165.

12. Liu Y, Wen F, Li J, Zuo C, Li M. Transitions of multifocal electroretinography in patients with age-related macular degeneration after combination therapy with photodynamic therapy and intravitreal bevacizumab. Doc Ophthalmol. 2009;119(3):163-169.
Clinical Ophthalmology

\section{Publish your work in this journal}

Clinical Ophthalmology is an international, peer-reviewed journal covering all subspecialties within ophthalmology. Key topics include: Optometry; Visual science; Pharmacology and drug therapy in eye diseases; Basic Sciences; Primary and Secondary eye care; Patient Safety and Quality of Care Improvements. This journal is indexed on

\section{Dovepress}

PubMed Central and CAS, and is the official journal of The Society of Clinical Ophthalmology (SCO). The manuscript management system is completely online and includes a very quick and fair peer-review system, which is all easy to use. Visit http://www.dovepress.com/ testimonials.php to read real quotes from published authors. 